ISSN No. 0974-035X

An indexed refereed \& peer-reviewed journal of higher education

Towards Excellence

UGC-HUMAN RESOURCE DEVELOPMENT CENTRE

Gujarat University, Ahmedabad-380009, Gujarat, India

\title{
સાહિત્ય અને આધ્યાત્મિક બુદ્ધિ
}

પ્રો. ડૉ. વીણાબેન કે. પટેલ

શ્રી અજય બી. રાવલ

\section{પ્રસ્તાવના}

જે વ્યક્તિ સાહિત્ય, સંગીત અથવા કળા વિનાની છે તે વ્યક્તિ પૂંછડા અને શીંગડા

વિનાના પશુ સમાન છે. આવા વ્યક્તિઓ ઘાસ નથી ખાતાં તે પેલાં પશુઓ ઉપર ઉપકાર છે. આ સંસ્કૃત સુભાષિતના ભાવાર્થમાં વ્યક્તિના જીવનમાં સાહિત્ય, સંગીત અને કળાનું કેટલું મહત્ત્વ રહેલું છે તે દર્શાવવામાં આવ્યું છે. માનવના ચોક્કસ વ્યક્તિત્વ ધડતર માટે આ ત્રણેય બાબત અથવા તે પૈકી કોઈ એક કે બે બાબતી ખૂબ મહત્ત્વનો ભાગ ભજવે છે. આ સુભાષિતમાં સુભાષિતકારે વ્યક્તિના જીવન માટે સાહિત્ય, સંગીત અને કળાની મહત્તા સૂચવી છે. અહીં, સાહિત્ય વ્યક્તિના બૌદ્ધિક વિકાસના પાસાને પ્રગટ કરે છે. સંગીત વ્યક્તિના મનને પ્રસન રાખી વિશ્વ પ્રત્યેના સૌહાર્દને પ્રગટાવે છે, જ્યારે કળા (કોઇપણ કળા) વ્યક્તિના ઇન્રિયગમ્ય કૌશલ્ય માટે જરૂરી છે. ત્રણ Hની આખી થિયરી કદાચ આ સૂક્તિમાંથી જ પ્રાપ્ત થઈજાય ! Head, Heart અને Handની સાથે અનુક્રમે સાહિત્ય, સંગીત અને કળાને ખૂબ સારી રીત સાંકળી શકાય છે. સાહિત્યનું અધ્યયન, શ્રવણ અને સ્વાધ્યાય મનને મોકળાશ આપે છે. સાહિત્યનો અભ્યાસ વ્યક્તિની બુદ્ધિને કુશાગ કરે છે. સાહિત્ય માત્ર બુદ્ધિને જ નહીં પરંતુ સંવેગોને પણ અભિવ્યક્ત કરવામાં સહાયક બને છે. સ્વામી વિવેકાનંદે ધર્મનું ચતિહાસિક વિશ્લેષણ કરતાં ભારતીયોની રાષ્ટ્રીય ‘આત્મા’ ધર્મને (અધ્યાત્મ) બતાવ્યો. આમ, ભારતીય સંસ્કૃતિમાં અધ્યાત્મનું આગવું 
મહત્ત્વ રહેલું છે. આધ્યાત્મિક બુદ્ધિ વ્યક્તિના સવાંગીણ આયામોના વિકાસનો માર્ગ પ્રશસ્ત કરે છે.

ચાવીરૂપ શબ્દોઃ સાહિત્ય, આધ્યાત્મિક બુદ્ધિ, આધ્યાત્મિકતા, ભાષા

\section{સાહિત્યની વ્યાખ્યાઓ}

સૌપ્રથમ આપણે “સાહિત્ય” શબ્દની વ્યુત્પત્તિ, અર્થ અને સંકલ્પનાને સમજીએ. સાહિત્ય

એક કળા છે. એટલું જ નહીં, સાહિત્ય સર્વોન્કૃષ્ટ કલાપ્રકાર છે.'સંસ્કૃતમાં ‘સાહિત્ય’શબ્દ સહિત પરથી બનેલો છે. 'સહિતત્વનો ભાવ' તે સાહિત્ય. ‘કાવ્ય' શબ્દ પણ સંસ્કૃતમાં 'સાહિત્ય'નો પર્યાય $छ$ छे.

આચાર્ય ભોજ 'શબ્દાર્થૌ સહિત્તી કાવ્યમ્’માં માને છે. તે સાહિત્યને આમ સમજાવે છ:"iિિં સાહિત્યમ્? યઃ શબ્દાર્થયોઃ સમબબન્ધઃ | અર્થાત્ સાહિત્ય શું છે? જેને શબ્દ અને અર્થ બજેની સાથે સંબંધ છે તે સાહિત્ય છે. તે સંબંધ બાર પ્રકારના હોય છે: ૧) અભિધા, ૨) વિવક્ષા, ૩ ) તાત્પર્યમ્, ૪) પ્રાવિભાગ:, ૫) વ્યપેક્ષા, ૬) સામર્થ્યમ, ૭) અન્વય:, ૮) એકાર્થી ભાવ:, ૯) દોષહાનમ,, ૧૦) ગુણોપાદાન, ૧ ૧ ) અલંકારયોગઃ, ૧ ૨) રસાવિયોગ:

આ પૈકી પ્રથમ આઠ પ્રકારના સંબંધ શબ્દ અને વાક્યની શક્તિના છે- વ્યાકરણ-ગત છે, છેલ્લા ચાર કાવ્યગત છે.

એબરક્રોમ્બી પ્રત્યાયનનો મહિમા કરતી વ્યાખ્યા આપે છે:

“સાહિત્યની કલા એટલે અનુભવને અનુભવ લેખે જ મૂલ્યવાન અને ઉપભોગ્ય ગણી ભાષા દ્વારા બીજાને પહોંચાડવાની કલા,'iii 
વિજયરાજ વૈદ્ય ‘સાહિત્યદર્શન'માં ‘સાહિત્ય એટલે શું?’ની ચર્ચા કરી તારવે છે, ‘સાહિત્ય એ પ્રતિભાવંત માનવ-આત્માની સિસૃક્ષાનો રસાત્મક કલાયુક્ત વાણીરૂપે આવિષ્કાર છે.'vત્તેમણ સાહિત્યમાં પ્રતિભા અને કલાનાં તત્ત્વોનો સમન્વય કર્યો છે.

\section{સાહિત્યનાં તત્ત્વો}

વિવિધ વ્યાખ્યાઓ પરથી ‘સાહિત્ય'માં કયાં કયાં તત્ત્વોનું 'સાહિત્ય'(સહિતપણું) સધાય છે તે નીચે મુજબ તારવી શકાય:

૧) શબ્દ અને અર્થનું 'સાહિત્ય’, ૨) સર્જક અને ભાવકનું ‘સાહિત્ય', ૩) સત્ય અને સૌંદર્યનું 'સાહિત્ય', ૪) સત્ય અને આનંદનું 'સાહિત્ય', ૫) ઊર્મિ, વિચાર અને કલ્પનાનું શબ્દગત 'સાહિત્ય', ૬) જીવન અને સાહિત્યનું ‘સાહિત્ય’.

આમ, સાહિત્ય એ જીવનના સત્યની સૌદર્યાત્મક, આનંદજનક, ભાષાગત, કલાત્મક અભિવ્યક્તિ છે.

\section{સાહિત્યના પ્રકાર}

સાહિત્યના જુદી-જુદી રીત વિભાગ પાડવામાં આવે છે જેમાં પાણિનીએ સાહિત્યના ઉદ્યવ પ્રમાણ: ૧)દષષ્ટ, ૨)પ્રોક્ત, ૩) ઉપજ્ઞાત, ૪) સૂત્ર, ૫) કૃત અને ૬) વ્યાખ્યાન એવા ભેદનો સમાવેશ થાય છે. આચાર્ય ભોજદેવે ૧) કાવ્ય, ૨) શાસ્ત્ર, ૩) ઇતિહાસ, ૪) કાવ્યશાસ્ત્ર, ૫) કાવ્યેતિહાસ અને ૬) શાસ્ત્રિતિહાસ એવા ભેદ કહ્યા છે. અન્યત્ર તણે ૧) વક્રકક્તિ, ૨) રસોક્તિ અને ૩) સ્વભાવોક્તિ એવા ત્રણ ભાગ કર્યા છ. 
પાશ્ચાત્ય સાહિત્યમીમાંસામાં હડસને સાહિત્યના વિષયો અને સાહિત્યપ્રેરક વૃત્તિઓન લક્ષમાં લઈને ત્રણ વર્ગી પાડ્યા છે: ૧) આત્માવિષ્ઠારનું સાહિત્ય, ૨) પરલક્ષી સાહિત્ય અને ૩) વર્ણનાત્મક સાહિત્ય. vi

ઉપરોક્ત તમામ વિભિજ આયામોથી પાડેલા ભેદને આધારે સાહિત્યના બે ભેદ સ્પષ્ટ પાડી શકાય છે: ૧) લલિત સાહિત્ય અને ૨) લલિતેતર સાહિત્ય. લલિત સાહિત્યમાં કાવ્ય, નવલકથા, વાર્તા, નાટક, સર્જનાત્મક લલિત નિબંધ વગેરેનો સમાવેશ થાય છે જ્યારે લલિતેતર સાહિત્યમાં માત્ર માહિતીપ્રચુર અને સર્જકતા વિનાના નિબંધો, લેખો, સંશોધનપત્રો, પ્રબંધો, આત્મકથા, રેખાચિત્ર, ચરિત્ર, પત્ર, ડાયરી વગેરેનો સમાવેશ થાય છે.vii

\section{આધ्यાત્મિક બુદ્ધિની વિભાવના}

પૂર્વના સમયમાં બુદ્ધિની સંકલ્પના કંઈક અલગ હતી. પહેલા તર્ક અને ગણિતમાં હેશિયાર હોય તેને જ બુદ્ધિમાન ગણવામાં આવતા હતાપરંતુ સમય જતા શ્રી હાવર્ડ ગાર્ડનરે બુદ્ધિની આ સંકલ્પનાનો વિસ્તાર કર્યો અને આઠ પ્રકારની બુદ્ધિનું અસ્તિત્વ તેમણે સ્વીકાર્યું, જેને "બહુવિધ બુદ્ધિ" નામ આપવામાં આવ્યું, પરંતુ આ આઠ પ્રકારની બુદ્ધિઓ પણ માત્ર ભૌતિક પરિપ્રેક્ષ્યમાં હતી. આ બુદ્ધિમાં સંવેગો અને લાગણીઓને ઓછું અથવા નહિવત્સ્થાન આપવામાં આવ્યુ હતુ. જેને ધ્યાનમાં રાખીન બુદ્ધિની સંકલ્પનાને વિસ્તારતાં શ્રી ડેનિયલ ગોલમેને “સાંવગિક બુદ્ધિ”ની સંકલ્પના આપી. કોઈપણ વ્યક્તિમાં બુદ્ધિ(IQ) અને સંવેગો(EQ) વત્તા-ઓછા પ્રમાણમાં હોય જ છે. પરંતુ યોગ્ય સમયે IQ અને EQનો ઉપયોગ જરૂરી છે. આમ, કયા સમયે કઈ બુદ્ધિ ઉપયોગમાં લેવી તે અથવા IQ અને EQને સમગતયા રીત “આધ્યાત્મિક બુદ્ધિ” કહે છે.ધર્મનું કેન્દ્ર તત્વ ‘અધ્યાત્મ' છે; અધ્યાત્મ ધર્મની પ્રાણ છે. કોઈપણ ધર્મ અધ્યાત્મ વિનાનો હોઈ ન શકે અને હોય તો ટકી ન શકે; અધ્યાત્મ વિનાનો ધર્મ આત્મા વિનાના દેશ જેવો છે. 
આધ્યાત્મિક બુદ્ધિના ચાર આયામો આ મુજબ છે૧૧) ઉચ્ય સ્વ-જાગૃકતા, ૨) વેશ્વિક જાગૃકતા, ૩) ઉચ્ય સ્વ-કુશળતા અને ૪) આધ્યાત્મિક હાજરી / સામાજિક કુશળતા ગાંધીજીએ આત્મકથાની પ્રસ્તાવનામાં લખ્યું છે કે,'મારે જે કરવું છે,જેની ફું 30 વર્ષ થયાં ઝંખના કરી રહ્યો છું, તે તો આત્મદર્શન છે, તે ઈશ્વરનો સાક્ષાત્કાર છે, મોક્ષ છે....

.... જે પ્રયોગો હંં કહેવાનો છું તે એવા નથી. તે પ્રયોગો આધ્યાત્મિક છે, અથવા તો નેતિક છે; આત્માની દૃષ્ટિએ પાળેલી નીતિ તે ધર્મ.'viiiઆનો અર્થ સ્પષ્ટ છે કે ગાંધીજીના જીવનનું કેન્દ્ર અધ્યાત્મ છે.

શ્રી રિચાર્ડ ગ્રિશ્સના મતે આધ્યાત્મિક બુદ્ધિ એટલે બુદ્ધિનું ઉચ્યતમ સ્તર કે જે આત્માની ક્ષમતા અને ગુણીને ડહાપણ, પ્રેમ, સર્જનશીલતા, શાંતિ અને ઊંડી અર્થગ્રણણ ક્ષમતાના સ્વરૂપમાં નિખારી દે છે, જેના વડે જરૂરી જીવન-કૌશલ્યો અને કાર્ય-કૌશલ્યોમાં સુધારો થાય છે.

આધ્યાત્મિક બુદ્ધિ એટલે માત્ર ધાર્મિક બાબતો જ નહિ. આધ્યાત્મિક બુદ્ધિના જુદા-જુદાં ધટકોમાં ભગવાન અને ધર્મ, ધાર્મિક સંકલ્પના, સામાજિક પરિપક્વતા, આધ્યાત્મિક જીવન વ્યવહાર (આધ્યાત્મિક બદલાવ), માનવતા, ખુલ્લાપણું, સંકલિતતા, દયા વગેરેની સમાવેશ થઈ શકે છે.

\section{સાહિત્ય અને આધ્યાત્મિક બુદ્ધિ}

સાહિત્ય અને આધ્યાત્મિક બુદ્ધિ વચ્ચેના સંબંધન નીચેના મુદ્દૌથી સમજીશું:

- સાહિત્ય પોતાની સાથે એક કાળખંડને સાથે રાખે છે. જે-તે સમયના સમાજની છબિ આપણે સાહિત્યમાં જોઈ શકીએ છીઓ. આમ તે સમયના જ્ઞાનને (ભલે કલ્પન તત્ત્વ સહિતના!) આપણે સાહિત્યની મદદથી સમજી શકીએ છીએ. આધ્યાત્મિક રીતે સમૃધ્ધ વ્યક્તિને જ્ઞાન પણ હોવું જરૂરી छे. 
- સાહિત્ય એ માનવના મનની લાગણીઓનું પ્રતિબિંબ હોય છે. સમાજના લોકોની સરેરાશ માનસિકતા શું છે તે જાણવામાં સાહિત્ય ઉપયોગી થઈ શકે છે. મનન જાણવાની બાબત આધ્યાત્મિક બુદ્ધિન સ્પર્શે છે.

- ઉત્તમ સાહિત્ય માનવીની ચેતનાન ઉર્ધ્વ ગતિ આપે છે. તેના થકી માનવી સ્વ-ચેતનામાંથી પરમ-ચેતના સુધીની યાત્રા સરળતાથી કરી શકે છે.

- સાહિત્ય માનવીના માનસિક વિકાસને વેગ આપે છે. મનના વિચારોની સમૃદ્ધિમાં શિષ્ટ સાહિત્ય ઉપયોગી બની શકે છે અને સાહિત્ય થકી ચિંતનનો વ્યાપ અને ક્ષેત્ર વિશાળ બનતું જાય છે.

- લલિત સાહિત્ય માનવીના સાંવગિક વિકાસમાં અને લલિતેતર સાહિત્ય માનવીના બૌદ્ધિક વિકાસમાં સહાયક બની શકે છે.

- શિષ્ટસાહિત્ય વાચક કેસર્જકના એક વિશાળ, વૈશ્चિક દૃષ્ટિકોણના નિર્માણનું કાર્ય કરી શકે છે.વ્યક્તિમાં રહેલા ઉત્તમ તત્વોના આવિષ્કરણ કે પ્રગટીકરણ માટે શિષ્ટ સાહિત્ય સહાય કરી શકે $छ$ छे.

- શિષ્ટસાહિત્ય થકી માણસ પોતાની લાગણીઓની અભિવ્યક્તિ કરી શકે છે જેનાથી વ્યક્તિનું હદદય કલુષિત થતું નથી, માણસ મૂંઝવણ અનુભવતો નથી.

- શિષ્ટસાહિત્યમાં રહેલા પાત્રો સાથે તાદાત્મ્ય જાળવીન વ્યક્તિ પોતાની સામાજિક, માનસિક અને આધ્યાત્મિક ઉન્ઞતિ કરી શકે છે.

- શિષ્ટસાહિત્ય એ મૂલ્ય સંવર્ધન થકી શ્રેષ્ઠ નાગરિકના નિર્માણમાં સહાયક બની શકે છે તમમજ આવી રીતે ધડાયેલ વ્યક્તિ પોતાના ક્ષેત્રમાં શ્રેષ્ઠ પ્રદાન આપી શકે છે.

- પોતપોતાના ક્ષેત્રમાં સફળ વ્યક્તિઓ પૈકી મોટાભાગના વ્યક્તિઓમાં વાંચનની ટેવ અવશ્યપણે જોવા મળશે. આ તેમની સફળતામાં સાહિત્યનો ફાળો જ ગણી શકાય. 
- માનસિક સ્વાસ્થ્યને જાળવવા માટે શિષ્ટસાહિત્ય ઉપયોગી બની શકે છે.જેવી રીતે શરીરના સૌષ્ઠવ માટે કસરત જરૂરી છે તેવી જ રીત મનના સ્વાસ્થ્ય માટે શિષ્ટ સાહિત્યનું વાચન ઉપયોગી બની રહે છે.

\section{ઉપસંહાર}

આમ, વ્યક્તિને સમાજને અનુફૂ બનાવવા, સવાંગી વિકાસના ધ્ચેયને પામવા તેમજ સમગતયા સર્વ માટે વિચારતો કરવાના સાધનોમાં સાહિત્યનો સમાવેશ થાય છે. સાહિત્ય થકી શ્રેષ્ઠ માનવીના પ્રગટીકરણની પ્રક્રિયા સાર્વત્રિક કરવી જોઈએ. 
Towards Excellence: An Indexed, Refereed \& Peer Reviewed Journal of Higher Education / Dr. Veenaben Patel \& Mr. Ajay Raval / Page 159-166

\section{સંદર્ભસૂચિ}

ંપટેલ, બહેચરભાઈ ર. (૧૯૯૨), સાહિત્યવિવેચન, યુનિવર્સિટી ગંથનિર્માણ બોર્ડ: અમદાવાદ, પૃ. ૧ iઉપરોક્ત, પૃ. ૨૭

iiiપારેખ, નગીનદાસ(અનુ. ), 'સાહિત્યવિવેચનના સિદ્ધાંતો', પૃ. ૪૭

iખપેલ, બહેચરભાઈ ૨. (૧૯૯૨), સાહિત્યવિવચન, યુનિવર્સિટી ગંથનિર્માણ બોર્ડ: અમદાવાદ, પૃ. $૩$ ૧

ચઉપરોક્ત, પૃ. ૩૬

viઉપપરોક્ત, પૃ. 39

viiઉપરોક્ત, પૃ. ૩૭-૩૮

viiiગાંધી, મોહનદાસ કરમચંદ; કુમારપપા, ભારતન(સંક્ષેપકાર), (૨૦૨૦), સંદ્ષિપ્ત આત્મકથા, ISBN- 978-81-

7229-353-6, નવજીવન ટ્રસ્ટ: અમદાવાદ

પ્રો. ડૉ. વીણાબેન કે. પટેલ

શ્રી અજય બી. રાવલ 